\title{
Study on the influence of the free volume of hybrid membrane on pervaporation performance by positron annihilation spectroscopy
}

\author{
Chi-Lan Li ${ }^{b}$, Shu-Hsien Huang ${ }^{a}$, Wei-Song Hung ${ }^{a}$, Se-Tsung Kao ${ }^{\text {a,c }}$, \\ Da-Ming Wang a,d, Y.C. Jean ${ }^{\text {a,e }}$, Kueir-Rarn Lee ${ }^{a, *}$, Juin-Yih Lai ${ }^{\text {a }}$ \\ ${ }^{a} R \& D$ Center for Membrane Technology, Department of Chemical Engineering, Chung Yuan University, Chung Li 32023, Taiwan \\ ${ }^{\mathrm{b}}$ Department of Chemical and Material Engineering, Nanya Institute of Technology, Chung Li 32034, Taiwan \\ ${ }^{\mathrm{c}}$ Institute of Polymer Science and Engineering, National Taiwan University, Taipei 10617, Taiwan \\ ${ }^{\mathrm{d}}$ Department of Chemical Engineering, National Taiwan University, Taipei 10617, Taiwan \\ e Department of Chemistry, University of Missouri Kansas City, Kansas City, MO 64110, USA \\ Received 14 November 2007; received in revised form 17 December 2007; accepted 21 December 2007 \\ Available online 5 January 2008
}

\begin{abstract}
To improve the pervaporation separation performance, an organic-inorganic hybrid membranes were prepared by adding zeolite $13 \mathrm{X}$ into the polyimide (BAPP-BODA), which was synthesized using one-step polycondensation polymerization of 2,2-bis(4-(4-aminophenoxy)phenyl)propane (BAPP) with bicyclo[2.2.2] oct-7-ene-2,3,5,6-tetracarboxylic dianhydride (BODA). The influence of the zeolite 13X content on the free volume and pervaporation performance of hybrid membranes were systematically analyzed by positron annihilation spectroscopy (PAS). The trend of orthopositronium $(o-\mathrm{Ps})$ lifetime and free volume holes changes consistently well with the permeation rate variation of aqueous isopropanol mixtures dehydration. The BAPP-BODA/13X hybrid membrane morphology was characterized by AFM and SEM. Compared with the BAPP-BODA membrane, the BAPP-BODA/13X hybrid membrane had good pervaporation performance for aqueous alcohol solution dehydration. The sorption selectivity dominates the behavior of pervaporation while adding zeolite $13 \mathrm{X}$ into the polyimide matrix.
\end{abstract}

(C) 2008 Elsevier B.V. All rights reserved.

Keywords: Polyimide; Zeolite; Positron annihilation spectroscopy; Pervaporation; Alcohol-water mixture

\section{Introduction}

Membrane pervaporation has become one of the most promising candidates for energy-saving, low-cost separation processes, especially for mixtures with close volatility, and mixtures with thermal or chemical sensitivity [1]. Applications can be found in (a) organic/water (azeotropic) mixture dehydration, (b) removal/recovery of organic compounds from water, and (c) separation of organic/organic mixtures. Pervaporation process is fundamentally based on differences in permeation speed in various substances or classes of substances through a given membrane. The transport mechanisms of various classes of substances through a pervaporation membrane can be described using a sorption-diffusion model [2]. According to this model, the transport of a given substance through a membrane takes

\footnotetext{
* Corresponding author. Tel.: +886 3 2654190; fax: +886 32654198 .

E-mail address: krlee@cycu.edu.tw (K.-R. Lee).
}

place within three consecutive processes: (1) sorption of the given substance onto the membrane, (2) transport through the membrane, and (3) desorption/evaporation from the membrane. The driving force for pervaporation is the pressure gradient that exists on opposite side of the membrane.

Reminiscent of the pervaporation performance of some glassy polymers, most of these materials exhibit a fairly high separation factor, albeit low permeation flux when separating aqueous alcohol mixtures. In an effort to increase the flux and/or improve the selectivity, many studies have incorporated metal complexes and zeolites in the membranes [3-6]. The zeolite-incorporated polymer membranes have received much attention recently in gas and pervaporation separation studies. The incorporation of zeolite or porous fillers in dense membranes can improve the separation performance [4-8] due to combined molecular sieving action, selective adsorption and difference in diffusion rates. In addition, zeolites have high mechanical strength, good thermal and chemical stability, and thus, these membranes can be used over the wide range of 

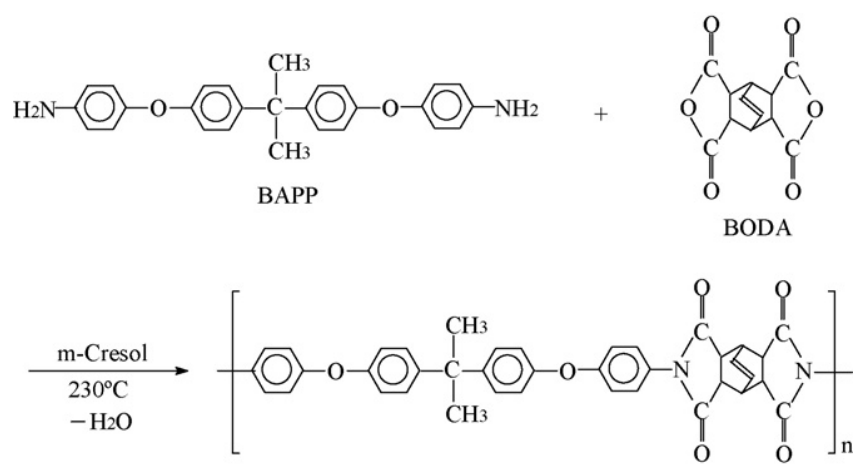

BAPP-BODA

Fig. 1. A synthesis scheme of the polyimide BAPP-BODA.

operating conditions when incorporated with these fillers. The zeolite 13X, Faujasite type, used in this study has a threedimensional pore structure. The pore entrances are composed of six-membered rings. The zoelite $13 \mathrm{X}$ particle and pore sizes are $2.67 \mu \mathrm{m}$ and $8.16 \AA$, respectively. The silicon/aluminum $(\mathrm{Si} / \mathrm{Al})$ ratio of zeolite $13 \mathrm{X}$ is 1.43 . The $\mathrm{Si} / \mathrm{Al}$ ratio indicates the hydrophilicity of zeolite $[9,10]$. In general, the hydrophilicity increases when the Si/Al ratio decreases. Thus, $13 \mathrm{X}$ can be considered hydrophilic, resulting from the lower $\mathrm{Si} / \mathrm{Al}$ ratio.

Polyimides have excellent thermal stability, good chemical resistance and mechanical strength. They have a low permeation rate when they are used as a membrane material for pervaporation separation. Hydrophilic zeolite 13X was introduced into the polyimide BAPP-BODA, which was synthesized using one-step polycondensation polymerization of 2,2-bis(4-(4-aminophenoxy)phenyl)propane (BAPP) with bicyclo[2.2.2] oct-7-ene-2,3,5,6-tetracarboxylic dianhydride (BODA). This was expected to improve the membrane pervaporation performance through the molecular sieving effect and hydrophilicity of the zeolite. Moreover, the influence of the zeolite $13 \mathrm{X}$ content on the free volume and pervaporation performance of hybrid membranes were systematically analyzed by positron annihilation spectroscopy (PAS).

\section{Experimental}

\subsection{Synthesis of polyimide}

The polyimide BAPP-BODA was prepared by one-step polymerization of the diamine, BAPP and the dianhydride, BODA. The synthetic route of the polyimide is illustrated in Fig. 1. A mixture of $30 \mathrm{mmol}$ of diamine BAPP, $30 \mathrm{mmol}$ of dianhydride BODA and $100 \mathrm{ml}$ of $m$-cresol was heated with stirring at $100^{\circ} \mathrm{C}$ for $1 \mathrm{~h}, 140^{\circ} \mathrm{C}$ for $1 \mathrm{~h}$, and $180^{\circ} \mathrm{C}$ for $1 \mathrm{~h}$. Then, the solvent was distilled at $230^{\circ} \mathrm{C}$, keeping the volume of the reaction mixture nearly constant by adding an appropriate volume of fresh $m$-cresol. The azeotropic removal of the water formed during the imidization was continued for $4 \mathrm{~h}$. The polymer solution was poured into a large amount of methanol. The precipitate, polyimide BAPP-BODA, was collected by filtration, washed thoroughly with methanol, and dried at $70^{\circ} \mathrm{C}$ under vacuum.
The inherent viscosity of the polymer was $0.6 \mathrm{dL} \mathrm{g}^{-1}$, measured in DMAc solution at $25^{\circ} \mathrm{C}$.

\subsection{Membrane preparation}

The dense polyimide membranes were prepared by casting a dichloromethane solution containing about $10 \mathrm{wt} \%$ polymer on glass plates. Membranes were then dried at the atmospheric temperature. After drying, the membranes were removed from the glass plates by distilled water, placed in a vacuum dryer and dried under vacuum at the atmospheric temperature for $24 \mathrm{~h}$. The zeolite 13X-filled polyimide membranes were prepared by adding an appropriate amount of zeolite $(13 \mathrm{X} / \mathrm{BAPP}-\mathrm{BODA}=0.25-0.75)$ into the $10 \mathrm{wt} \%$ polymer solution (BAPP-BODA/dichloromethane). The following steps were the same with the preparation of the dense polyimide membranes. The resulting polyimide and zeolite 13X-filled polyimide membranes had a thickness of $48-60 \mu \mathrm{m}$.

\subsection{Characterization of $B A P P-B O D A / 13 X$ hybrid membrane}

The cross-sectional morphology of membranes was observed with scanning electron microscope (SEM). The surface morphology of the membrane was observed with an AFM (Digital Instruments, DI-NS3a USA). In order to investigate the variation of the free volume in the BAPP-BODA/13X hybrid membrane, the positron annihilation spectroscopy (PAS) experiments were performed. The positron annihilation spectroscopy of BAPP-BODA/13X hybrid membranes were determined by detecting the prompt $\gamma$-rays $(1.28 \mathrm{MeV})$ from the nuclear decay that accompanies the emission of a positron from the ${ }^{22} \mathrm{Na}$ radioisotope and the subsequent annihilation $\gamma$-rays $(0.511 \mathrm{MeV})$. All of the PAL spectra were analyzed by a finiteterm lifetime analysis method using the PATFIT program and a continuous lifetime distributions using the MELT program. It had been reported in our previous paper [11,12]. We employ the results of $o$-Ps lifetime to obtain the volume of free volume holes.

\subsection{Pervaporation study}

A traditional pervaporation process was used [13]. The effective membrane area was $11.6 \mathrm{~cm}^{2}$ and the feed temperature studied was $25^{\circ} \mathrm{C}$. The permeation rate was determined by measuring the weight of the permeate. The compositions of the feed solutions, the permeates, and the solutions adsorbed in the membrane were measured by gas chromatography (GC China Chromatography 8700T). The separation factor $\left(\alpha_{\text {water/alcohol }}\right)$ was calculated as follows:

$\alpha_{\text {water } / \text { alcohol }}=\frac{Y_{\text {water }} / Y_{\text {alcohol }}}{X_{\text {water }} / X_{\text {alcohol }}}$

where $X_{\text {water }}, X_{\text {alcohol }}$ and $Y_{\text {water }}, Y_{\text {alcohol }}$ are the weight fraction of water and alcohol in the feed and permeate, respectively. 


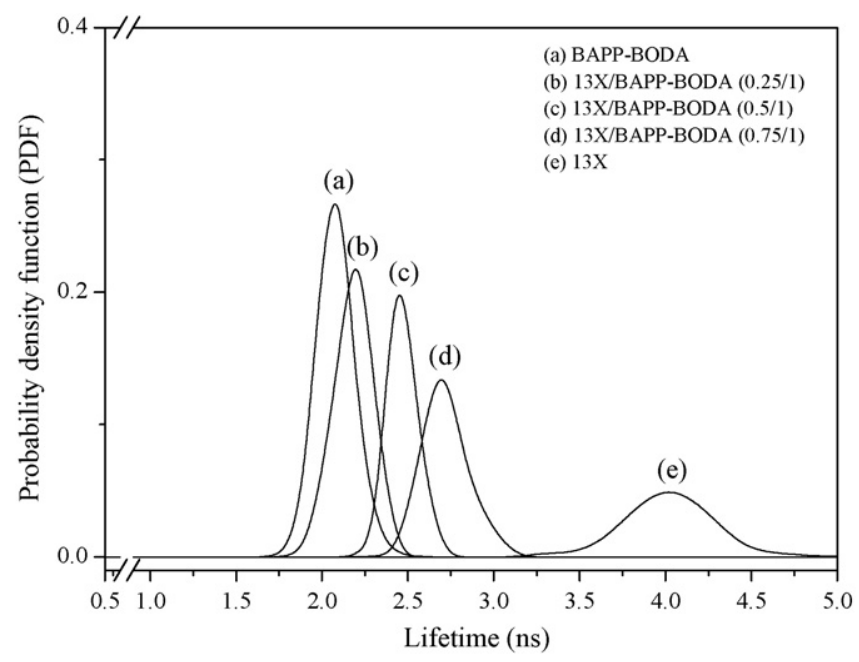

Fig. 2. $o$-Ps lifetime distributions of the BAPP-BODA/13X hybrid membranes.

\subsection{Sorption measurement}

The membranes were immersed in several feed mixtures for $24 \mathrm{~h}$ at $25^{\circ} \mathrm{C}$. Then, they were erased between pieces of filter paper to remove excess solution and quickly placed in the left half of a twin-tube setup cooled with liquid nitrogen. The system was evacuated while the left tube was heated with hot water and the right tube was cooled in liquid nitrogen. GC determined the composition of the condensed liquid in the right tube. The separation factor of sorption $\left(\alpha_{\text {sorp }}\right)$ was calculated as follows:

$\alpha_{\text {sorp }}=\frac{Y_{\text {water }} / Y_{\text {alcohol }}}{X_{\text {water }} / X_{\text {alcohol }}}$

where $X_{\text {water }}, X_{\text {alcohol }}$ and $Y_{\text {water }}, Y_{\text {alcohol }}$ are the weight fraction of water and alcohol in the feed and membrane, respectively.

\subsection{Degree of swelling}

The membranes were immersed in different feed mixtures for $24 \mathrm{~h}$ at $25^{\circ} \mathrm{C}$. The degree of swelling of the membrane was defined by the following equation:

degree of swelling $=\frac{W_{\mathrm{w}}-W_{\mathrm{d}}}{W_{\mathrm{d}}} \times 100 \%$

where $W_{\mathrm{d}}$ and $W_{\mathrm{w}}$ denote the weight of dry and swollen membranes, respectively.

\section{Results and discussion}

\subsection{Characterization of the $B A P P-B O D A / 13 X$ hybrid membrane}

To understand the free volume size distribution of BAPP-BODA/13X hybrid membranes, the position lifetime distributions and the $\tau_{3}$ results are analyzed through the positron annihilation spectroscopy, as shown in Fig. 2. We employ the results of $o$-Ps annihilation lifetime $\left(\tau_{3}\right)$ to analyze the volume of free volume hole. Fig. 2 shows the effect of zeolite $13 \mathrm{X}$ content of the BAPP-BODA/13X hybrid membranes on

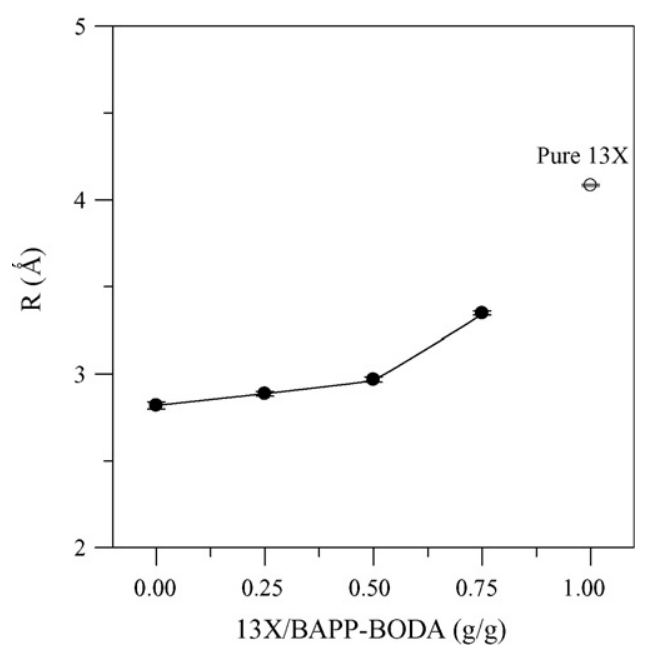

Fig. 3. Effect of zeolite $13 \mathrm{X}$ content on free volume hole radius $(R)$.

the $o$-Ps annihilation lifetime. It shows that the $o$-Ps annihilation lifetime increases with increasing the zeolite $13 \mathrm{X}$ content of the BAPP-BODA/13X hybrid membranes. This result indicates that the size of free volume hole is strongly composition dependent. On the other hand, the lifetime distribution curves shift to higher lifetime direction, as the zeolite $13 \mathrm{X}$ content increases. These phenomena might be due to the fact that the free volume radius of pure BAPP-BODA and zeolite $13 \mathrm{X}$ particle is 2.85 and $4.08 \AA$, respectively. Thus, the free volume radius of the BAPP-BODA/13X hybrid membranes lie in between the pure BAPP-BODA and zeolite $13 \mathrm{X}$ particle, as shown in Fig. 3. Another interesting phenomena revealed in this positron annihilation spectroscopy is that the relative intensity of $o$-Ps annihilation lifetime $\left(I_{3}\right)$ decreases with increasing the zeolite 13X content (Fig. 4). The decrease in the relative intensity of $o$-Ps annihilation lifetime $\left(I_{3}\right)$ suggests that the adding zeolite $13 \mathrm{X}$ particle into the BAPP-BODA polyimide matrix reduces the number of free volume holes. However, the volume of free volume holes (with corresponding $o$-Ps annihilation lifetime, $\tau_{3}$ ) increases by increasing the zeolite $13 \mathrm{X}$ content of the BAPP-BODA/13X hybrid membranes, also shown in Fig. 5.

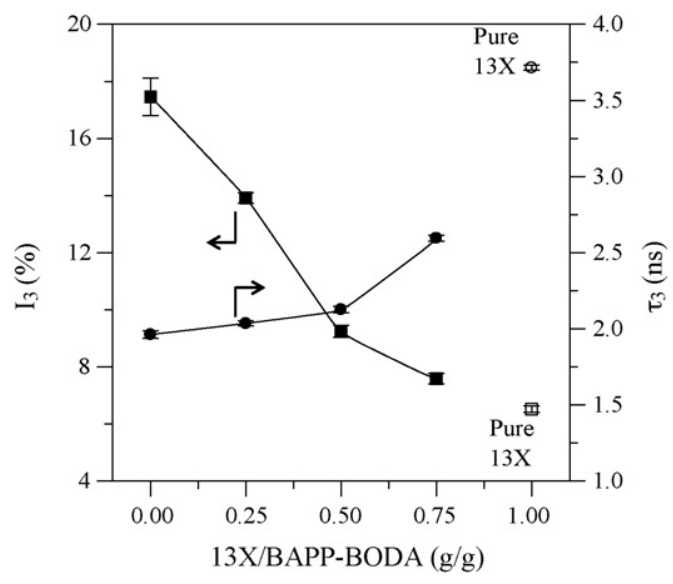

Fig. 4. Effect of zeolite $13 X$ content on $(\square)(\square)$ intensity $\left(I_{3}\right)$ and $(\bigcirc)(\bigcirc)$ lifetime $\left(\tau_{3}\right)$ 


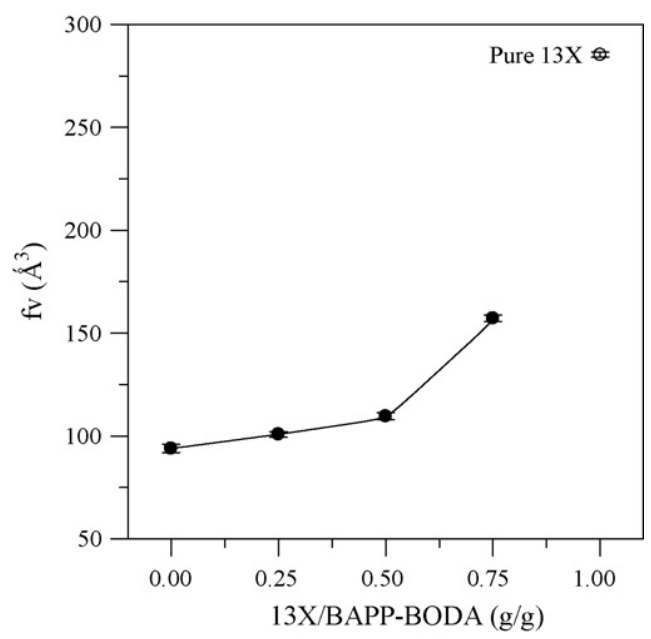

Fig. 5. Effect of zeolite 13X content on volume of free volume hole (fv).

These phenomena imply that the interfacial voids formed, resulting from the incompatibility between polyimide matrix and zeolite particles as well as the hole volume of zeolite particles. It also causes the distribution of free volume hole size shifts from smaller size to larger size in the BAPP-BODA/13X hybrid membranes (corresponding with Fig. 2).

\subsection{Effect of zeolite content on the pervaporation performance}

The zeolite content effect on the BAPP-BODA/13X hybrid membranes in the pervaporation of a $90 \mathrm{wt} \%$ aqueous isopropanol mixture is shown in Fig. 6. It shows that the permeation rate increases with increasing zeolite content in the BAPP-BODA/13X hybrid membrane. This phenomenon might be due to the molecular sieving effect and hydrophilicity of the zeolite 13X introduced into the polyimide membrane. From the analysis of positron annihilation spectroscopy, the volume of free volume holes increases by increasing the zeolite $13 \mathrm{X}$ content of the BAPP-BODA/13X hybrid membranes (Fig. 5). These results correspond well with the results mentioned above, as

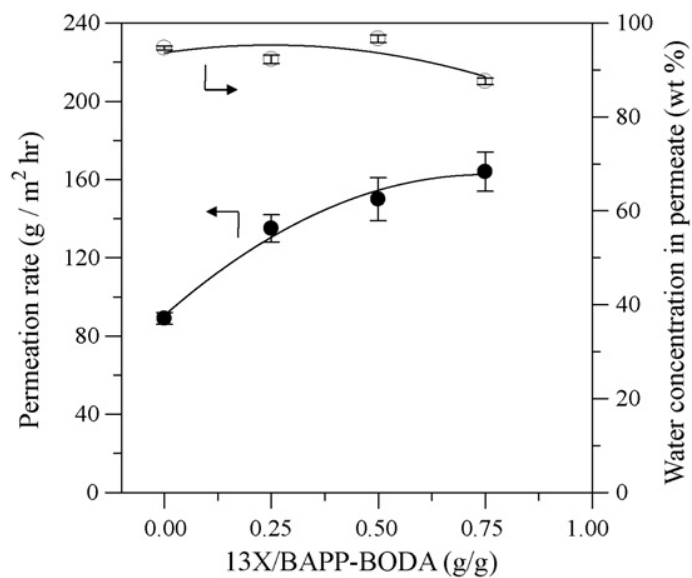

Fig. 6. Effect of zeolite 13X content on pervaporation separation of $90 \mathrm{wt} \%$ aqueous isopropanol mixture for the BAPP-BODA/13X hybrid membranes at $25^{\circ} \mathrm{C}$.

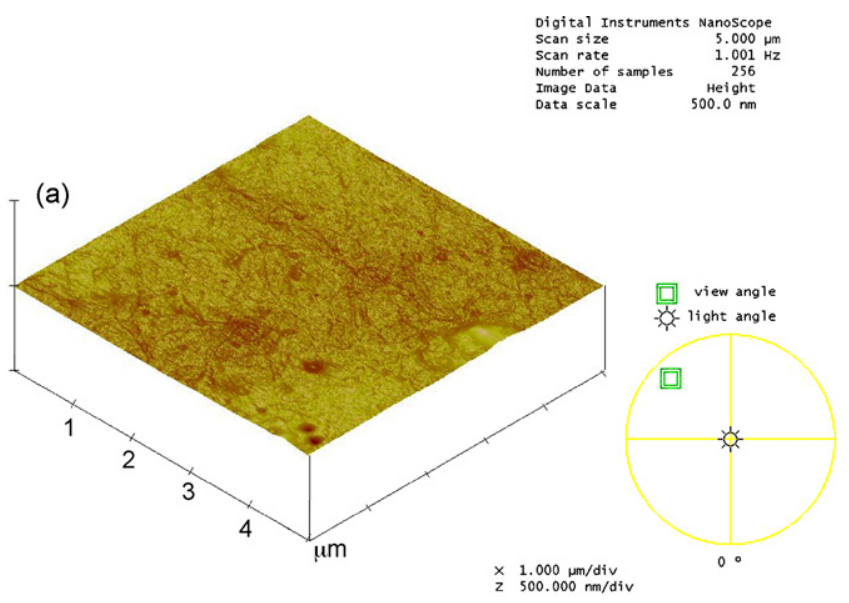

pi -002.000

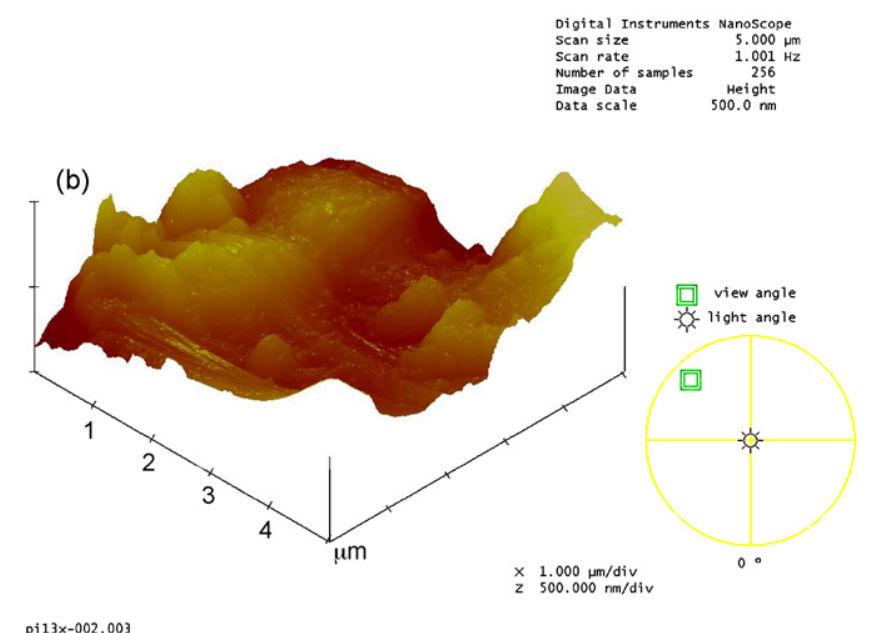

Fig. 7. AFM images of the polyimide membranes: (a) BAPP-BODA and (b) BAPP-BODA/13X $(13 X /$ BAPP-BODA $=0.5 / 1(\mathrm{~g} / \mathrm{g}))$.

indicated in Fig. 6. In general, a rougher membrane surface can improve the affinity to water, resulting in an increased permeation rate. To investigate the membrane surface morphology, AFM studies are conducted, as shown in Fig. 7. The surface roughness for the BAPP-BODA membrane is lower than that of the BAPP-BODA/13X hybrid membrane. As a result, the effective pervaporation membrane area increases with increasing membrane roughness. These results also correspond well with the results indicated in Fig. 6. Moreover, a cross-section image (Fig. 8) of the zeolite 13X-filled polyimide membrane with a zeolite/polyimide ratio of $0.5 / 1$ by weight shows that many interfacial voids appear around the zeolite particles. The hard segment of the polyimide chains might cause the interfacial voids, that is, the polyimide chains might be too stiff to wrap around the zeolite well. Therefore, the permeation rate increases with increasing zeolite content in the BAPP-BODA/13X hybrid membrane. Fig. 6 also shows that the water concentration in the permeate almost unchanges while the zeolite content in the BAPP-BODA/13X hybrid membrane increases. These phenomena might be because the degree of swelling decreases, as 


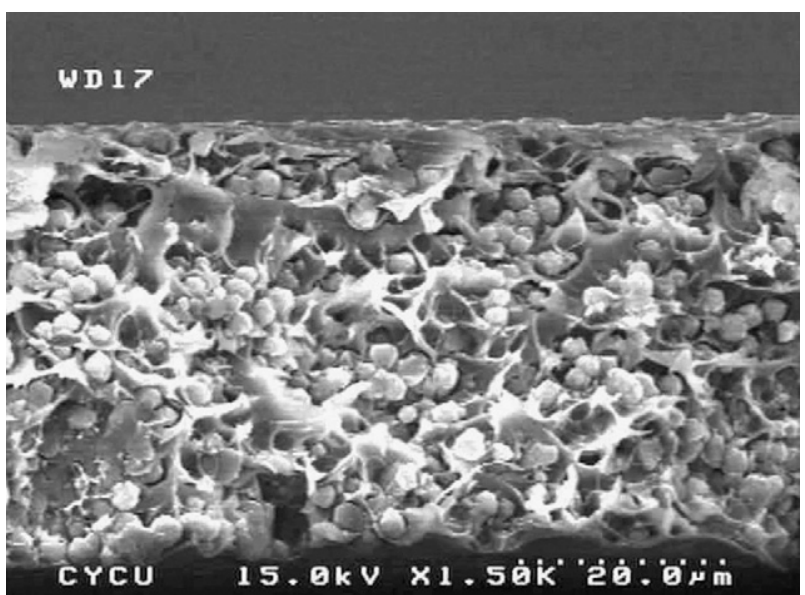

Fig. 8. Cross-section morphology of the BAPP-BODA/13X hybrid membrane: 13X/BAPP-BODA $=0.5 / 1(\mathrm{~g} / \mathrm{g})$.

shown in Fig. 9, by incorporating zeolite $13 \mathrm{X}$ into the polyimide membrane to inhibit polymer chain mobility and increase resistance to the larger permeates, isopropanol, through the BAPP-BODA/13X hybrid membrane. In the past, the selectivity (corresponding with water concentration in the permeate) of pervaporation separation process has been traditionally understood in terms of the interaction between the permeates and the polymeric membranes. Recently, positron annihilation spectroscopy studies could provide a new approach in terms of size selectivity of free volume. Fig. 3 also shows the results of the free volume hole radii of hybrid membranes. It is very interesting that the free volume radius distribution of the BAPP-BODA/13X hybrid membranes lies between the kinetic radius of water $(1.2 \AA)$ and isopropanol $(3.86 \AA)$. Thus, the water concentration in the permeate almost unchanges while the zeolite content in the BAPP-BODA/13X hybrid membrane increases.

\subsection{Pervaporation of alcohol-water mixtures through $B A P P-B O D A / 13 X$ hybrid membrane}

Table 1 shows the pervaporation performance of $90 \mathrm{wt} \%$ alcohol-water mixtures through the BAPP-BODA/13X hybrid membrane. The permeation rate of both polyimide membranes decreases, but the water concentration in the permeate increases with increasing alcohol molar volume. The alcohol molar volumes can explain these phenomena. The molar volumes of methanol, ethanol, isopropanol, and $n$-butanol are 40.7, 58.68,

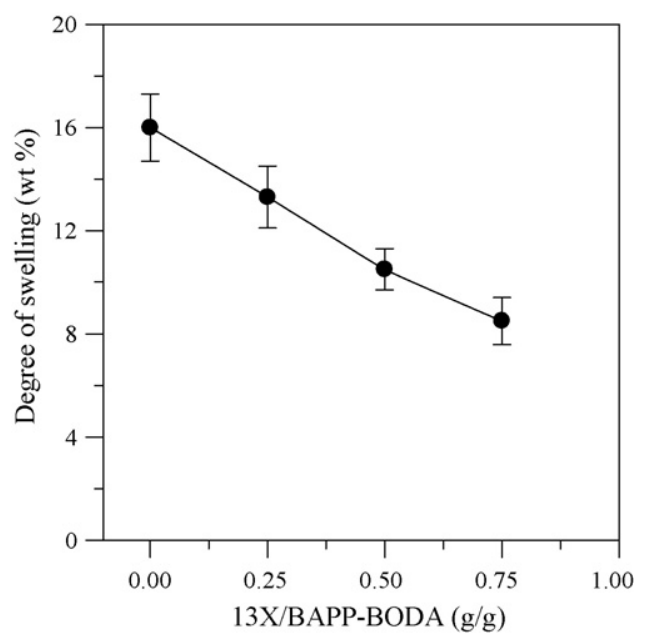

Fig. 9. Effect of zeolite $13 \mathrm{X}$ content on degree of swelling of $90 \mathrm{wt} \%$ aqueous isopropanol mixture for the BAPP-BODA/13X hybrid membranes at $25^{\circ} \mathrm{C}$.

75.14 , and $94.88 \mathrm{ml} / \mathrm{mol}$, respectively. The water concentration in the permeate is found to depend on the molecular length of linear alcohols. In addition, the permeation rate of $n$-butanol is higher than that of isopropanol. It is possibly because the steric hindrance of the former is lower than that of the latter. A higher water concentration in the permeate and a lower permeation rate are achieved for a higher molecular weight alcohol. Compared with the BAPP-BODA polyimide membrane, the BAPP-BODA/13X hybrid membrane has a higher permeation rate with similar water concentrations in the permeate. This is possibly due to the molecular sieving effect and hydrophilicity of the zeolites. These results correspond well with the position lifetime distributions and the $\tau_{3}$ results indicated in Figs. 2 and 4 . The positron annihilation lifetime $\left(\tau_{3}\right)$ of the BAPP-BODA polyimide is lower than that of the BAPP-BODA/13X hybrid membrane. Sorption experiments are conducted to investigate the solubility and diffusivity effects on the permselectivity of the BAPP-BODA/13X hybrid membranes. Fig. 10 shows the alcohol concentration effect on the water concentration in the membrane. It is found that the water concentration in the BAPP-BODA/13X hybrid membrane is higher than that for the BAPP-BODA polyimide membrane. These results might be due to the hydrophilicity of the zeolite. As the carbon atomic number of the alcohol increases, the water concentration in the BAPP-BODA polyimide membrane decreases rapidly and that in the BAPP-BODA/13X hybrid membrane increases slightly.

Table 1

Results on the pervaporation separation performance of aqueous alcohol mixtures through the pristine BAPP-BODA and BAPP-BODA/13X hybrid membranes ${ }^{\mathrm{a}}$

\begin{tabular}{|c|c|c|c|c|c|c|}
\hline \multirow[t]{2}{*}{ Aqueous alcohol mixtures (90 wt\%) } & \multicolumn{3}{|c|}{ BAPP-BODA } & \multicolumn{3}{|c|}{ BAPP-BODA/13X } \\
\hline & $\mathrm{A}\left(\mathrm{g} / \mathrm{m}^{2} \mathrm{~h}\right)$ & $\mathrm{B}(\mathrm{wt} \%)$ & $\mathrm{C}(\%)$ & $\mathrm{A}\left(\mathrm{g} / \mathrm{m}^{2} \mathrm{~h}\right)$ & $\mathrm{B}(\mathrm{wt} \%)$ & $\mathrm{C}(\%)$ \\
\hline Methanol & 238 & 24.1 & 5.5 & 320 & 22.2 & 4.5 \\
\hline Ethanol & 152 & 57.2 & 12.3 & 210 & 48.3 & 6.1 \\
\hline Isopropanol & 90 & 94.6 & 16.2 & 150 & 96.8 & 10.2 \\
\hline n-Butanol & 145 & 95.8 & 21.4 & 190 & 96.7 & 13.5 \\
\hline
\end{tabular}

A: permeation rate $\left(\mathrm{g} / \mathrm{m}^{2} \mathrm{~h}\right)$; B: water concentration in permeate $(\mathrm{wt} \%)$; C: degree of swelling $(\%)$; operation temperature: $25^{\circ} \mathrm{C}$.

${ }^{a}$ BAPP-BODA/13X hybrid membrane composition: 13X/BAPP-BODA = 0.5/1 (g/g). 
Table 2

Solution-diffusion analysis of the pristine BAPP-BODA and BAPP-BODA/13X hybrid membranes ${ }^{\mathrm{a}}$

\begin{tabular}{|c|c|c|c|c|c|c|}
\hline Aqueous alcohol mixtures (90 wt $\%$ ) & \multicolumn{3}{|c|}{ BAPP-BODA } & \multicolumn{3}{|c|}{ BAPP-BODA/13X } \\
\hline Methanol & 2.8 & 28.5 & 0.01 & 2.5 & 66.2 & 0.04 \\
\hline Isopropanol & 157.6 & 4.8 & 32.5 & 272.5 & 171.4 & 1.58 \\
\hline$n$-Butanol & 202.5 & 2.2 & 91.2 & 263.7 & 141.2 & 1.87 \\
\hline
\end{tabular}

$\alpha$ : overall selectivity; $\alpha_{S}$ : solution selectivity; $\alpha_{D}$ : diffusion selectivity; operation temperature: $25^{\circ} \mathrm{C}$.

a BAPP-BODA/13X hybrid membrane composition: 13X/BAPP-BODA = 0.5/1 (g/g).

The decreasing water concentration in the BAPP-BODA polyimide membrane is due to increasing degree of swelling, as shown in Table 1. The increasing water concentration in the BAPP-BODA/13X hybrid membrane results from the increased hydrophilicity from zeolite $13 \mathrm{X}$ incorporation into the polyimide membrane.

Pervaporation membranes generally follow the solution-diffusion model. According to the solution-diffusion model, the permeability $(P)$ is determined by the solubility $(S)$ and the diffusivity $(D)$. Therefore, the overall selectivity is determined by both the solution and diffusion selectivities; i.e. $P=S D$, and $\alpha_{\text {water/alcohol }}=P_{\text {water }} / P_{\text {alcohol }}=\left(S_{\text {water }} / S_{\text {alcohol }}\right)$ $\left(D_{\text {water }} / D_{\text {alcohol }}\right)=\alpha_{S} \alpha_{D}$. The solubility $S$ is obtained using sorption measurements. The diffusion $D$ is calculated from the $P$ to $S$ ratio. Table 2 shows the molar volume effect of aqueous alcohol mixtures on overall selectivity $(\alpha)$, solution selectivity $\left(\alpha_{S}\right)$ and diffusion selectivity $\left(\alpha_{D}\right)$ for the pristine BAPP-BODA and BAPP-BODA/13X hybrid membranes. It shows that $\alpha$ and $\alpha_{D}$ increase but $\alpha_{S}$ decreases with increasing alcohol carbon atomic number of feed mixtures for the BAPP-BODA polyimide membrane. Obviously, the diffusion selectivity dominates the pervaporation behavior. However, by incorporating zeolite 13X into the polyimide membrane, the variation of $\alpha$ corresponds with $\alpha_{S}$ with increasing alcohol carbon atomic number. This means that the sorption selectivity dominates the behavior of pervaporation because the separation factor of pervaporation

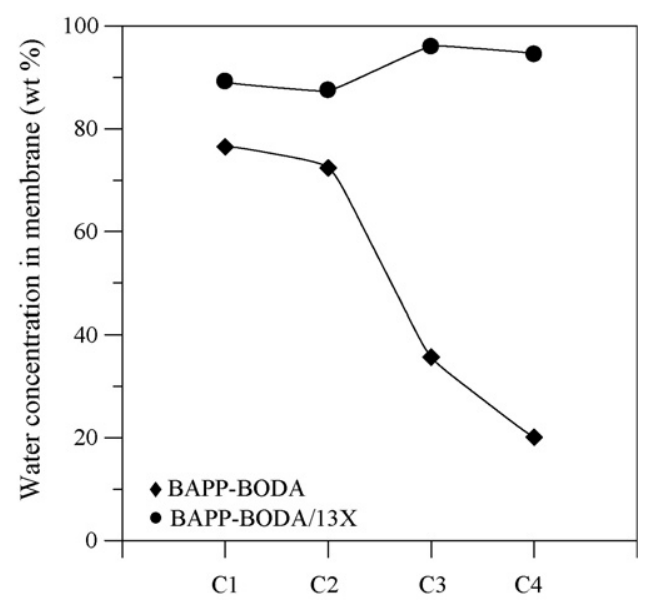

Fig. 10. Sorption experiments of $90 \mathrm{wt} \%$ alcohol-water mixtures at $25^{\circ} \mathrm{C}$ : $(\downarrow)$ BAPP-BODA; (-) BAPP-BODA/13X (13X/BAPP-BODA = 0.5/1 (g/g)). C1: methanol; C2: ethanol; $\mathrm{C} 3$ : isopropanol; $\mathrm{C} 4:$-butanol.

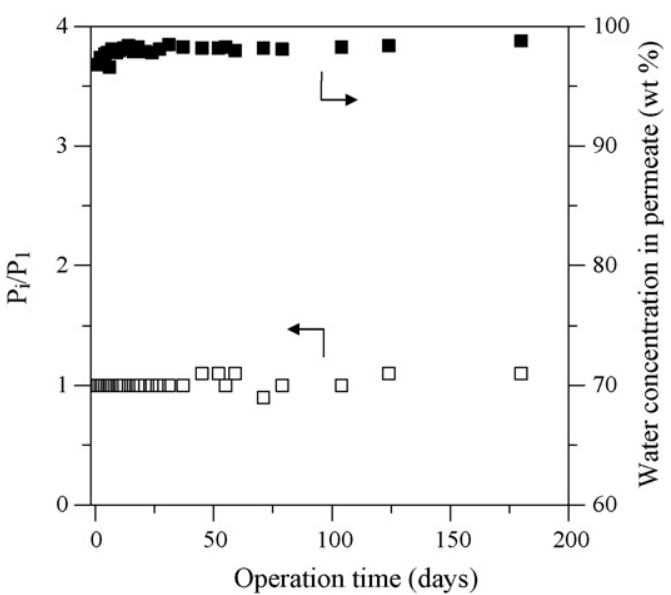

Fig. 11. Long-term experiment results for pervaporation separation of $70 \mathrm{wt} \%$ aqueous isopropanol mixture through the BAPP-BODA/13X hybrid membrane $(13 \mathrm{X} / \mathrm{BAPP}-\mathrm{BODA}=0.5 / 1(\mathrm{~g} / \mathrm{g}))$ at $25^{\circ} \mathrm{C}$. $(\square) P_{i} / P_{1}$ (the permeation rate ratio of the $i$ th day to the 1 st day); ( $\mathbf{\square})$ water concentration in permeate.

through the BAPP-BODA/13X hybrid membranes follows the same trend as the sorption selectivity but not the trend of the diffusion selectivity. The increasing $\alpha_{S}$ is due to incorporating the hydrophilic, larger pore size $(8.16 \AA)$ zeolite $13 \mathrm{X}$. Zeolite $13 \mathrm{X}$ dominates the overall selectivity for the BAPP-BODA/13X hybrid membranes.

\subsection{Durability of BAPP-BODA/13X hybrid membrane}

Long-term membrane stability experiments are performed in this study. Fig. 11 shows the durability of the BAPP-BODA/13X hybrid membrane for a $70 \mathrm{wt} \%$ aqueous isopropanol mixture at $25^{\circ} \mathrm{C}$. It shows that the water concentration in the permeate and the $P_{i} / P_{1}$ ratio (the permeation rate ratio of the $i$ th day to the 1st day) show no evident change during the long-term test procedure. It exhibits that BAPP-BODA/13X has good pervaporation performance although incompatibility exists between the polyimide matrix and zeolite particles. The pervaporation performance of a $70 \mathrm{wt} \%$ isopropanol-water mixture at $25^{\circ} \mathrm{C}$ is maintained for at least 180 days for the BAPP-BODA/13X hybrid membrane.

\section{Conclusions}

In the past, the selectivity of pervaporation separation process has been traditionally understood in terms of the interaction 
between the permeates and the polymeric membranes. Recently, positron annihilation spectroscopy studies could provide a new approach in terms of size selectivity of free volume. By incorporating zeolite $13 \mathrm{X}$ into the polyimide membrane, the $o$-Ps annihilation lifetime increases with increasing the zeolite $13 \mathrm{X}$ content of the BAPP-BODA/13X hybrid membranes. This result indicates that the size of free volume hole is strongly composition dependent. Compared with the BAPP-BODA membrane, the BAPP-BODA/13X hybrid membrane has good pervaporation performance for dehydrating aqueous alcohol solutions. In the long-term stability examination, the pervaporation of a $70 \mathrm{wt} \%$ isopropanol-water mixture at $25^{\circ} \mathrm{C}$ could be maintained for at least 180 days for the BAPP-BODA/13X hybrid membrane.

\section{Acknowledgements}

The authors wish to sincerely thank the Ministry of Economic Affairs, Ministry of Education Affair and the National Science Council of Taiwan, for financially supporting this work.

\section{References}

[1] P. Shao, R.Y.M. Huang, Polymeric membrane pervaporation, J. Membr. Sci. 287 (2007) 162-179.

[2] M.H.V. Mulder, A. Smolders, Pervaporation, solubility aspects of the solution-diffusion model, Sep. Purif. Methods 15 (1986) 119.

[3] K.J. Kim, S.H. Park, W.W. So, S.J. Moon, Pervaporation separation of aqueous organic mixtures through sulfated zirconia-poly(vinyl alcohol) membrane, J. Appl. Polym. Sci. 79 (2001) 1450-1455.
[4] X. Chen, Z.H. Ping, Y.C. Long, Separation properties of alcohol-water mixture through silicalite-i-filled silicone rubber membranes by pervaporation, J. Appl. Polym. Sci. 67 (1998) 629-636.

[5] X.M. He, W.H. Chan, C.F. Ng, Water-alcohol separation by pervaporation through zeolite-modified poly(amidesulfonamide), J. Appl. Polym. Sci. 82 (2001) 1323-1329.

[6] A.A. Kittur, M.Y. Kariduraganavar, U.S. Toti, K. Ramesh, T.M. Aminabhavi, Pervaporation separation of water-isopropanol mixtures using ZSM-5 zeolite incorporated poly(vinyl alcohol) membranes, J. Appl. Polym. Sci. 90 (2003) 2441-2448.

[7] M.Y. Kariduraganavar, A.A. Kittur, S.S. Kulkarni, K. Ramesh, Development of novel pervaporation membranes for the separation of water-isopropanol mixtures using sodium alginate and $\mathrm{NaY}$ zeolite, J. Membr. Sci. 238 (2004) 165-175.

[8] S.S. Kulkarni, S.M. Tambe, A.A. Kittur, M.Y. Kariduraganavar, Preparation of novel composite membranes for the pervaporation separation of water-acetic acid mixtures, J. Membr. Sci. 285 (2006) 420-431.

[9] M.Y. Teng, K.R. Lee, S.C. Fan, D.J. Liaw, J. Huang, J.Y. Lai, Development of aromatic polyamide membranes for pervaporation and vapor permeation, J. Membr. Sci. 164 (2000) 241-249.

[10] C.F. Mellot, A.K. Cheetham, S. Harms, S. Savitz, R.J. Gorte, A.L. Myers, Calorimetric and computational studies of chlorocarbon adsorption in zeolites, J. Am. Chem. Soc. 120 (1998) 5788-5792.

[11] C.C. Hu, Y.J. Fu, S.W. Hsiao, K.R. Lee, J.Y. Lai, Effect of physical aging on the gas transport properties of poly(methyl methacrylate) membranes, J. Membr. Sci. 303 (2007) 29-36.

[12] H.M. Chen, W.S. Hung, C.H. Lo, S.H. Huang, M.L. Cheng, G. Liu, K.R Lee, J.Y. Lai, Y.M. Sun, C.C. Hu, R. Suzuki, T. Ohdaira, Y.C. Jean, Free-volume depth profile of polymeric membranes studied by positron annihilation spectroscopy: layer structure from interfacial polymerization, Macromolecules 40 (2007) 7542-7557.

[13] M. Sakuth, J. Meyer, J. Gmehling, Vapor phase adsorption equilibria of toluene + 1-propanol mixtures on y-zeolites with different silicon to aluminum ratios, J. Chem. Eng. Data 40 (1995) 895-899. 